

\title{
The behavioral and molecular effects of estradiol and progesterone on a rat model of spinothalamic tract lesion
}

Abbas Alimoradian $^{1}$, Fatemeh Abbaszadeh ${ }^{2}$, Masoumeh Jorjani2, ${ }^{2 *}$ (D), Mehdi Sadegh ${ }^{4}$

\author{
1. Department of Pharmacology, School of Medicine, Arak University of Medical Sciences, Arak, Iran \\ 2. Neurobiology Research Center, Shahid Beheshti University of Medical Sciences, Tehran, Iran \\ 3. Department of Pharmacology, School of Medicine, Shahid Beheshti University of Medical Sciences, Tehran, Iran \\ 4. Department of Physiology, School of Medicine, Arak University of Medical Sciences, Arak, Iran
}

\begin{abstract}
Introduction: The spinal cord injury is temporary or permanent damage in the spinal cord that disturbs the motor and sensory functions. The neuroprotective effects of steroids has been reported previously. Therefore, we designed a study to investigate the effects of different doses of estradiol (Est) and progesterone (Prog) on unilateral lesion of the spinothalamic tract (STT).

Methods: The 77 male adult Wistar rats were under the anesthesia for dorsal laminectomy at the spinal segments T8-T9. A tungsten-electrode was targeted to the right STT and unilateral lesion was made by a brief current pulse $(300 \mu \mathrm{A}, 90 \mathrm{~s})$. Rats were divided into 11 groups and Est or Prog $(2,4,8$ and $16 \mathrm{mg} / \mathrm{kg})$ were administered 30min post-injury. Mechanical allodynia and open field as assessed before, 14 and 28 days after the injection then the animals were sacrificed. The western blotting was performed on T8-9 spinal segments to evaluate protein expression of ERK, p-P38, JNK, Iba1 and GFAP at the lesion site.

Results: Est but not Prog significantly increased the pain threshold and motor activity at the dose of $8 \mathrm{mg} / \mathrm{kg}$ on post-surgery days 14 and 28 . Est but not Prog significantly increased the protein expression of ERK while decreased JNK protein. Both Est and Prog significantly decreased protein expression of p-P38, Iba1 and GFAP.

Conclusion: These results show Est $(8 \mathrm{mg} / \mathrm{kg})$ is able to decrease mechanical allodynia and improve motor activity 14 and 28 days after spinothalamic tract lesion. It seems ERK, p-P38, JNK, Iba1 and GFAP are involved.
\end{abstract}

\author{
Keywords: \\ Estradiol \\ Progesterone \\ Spinal cord injury \\ Neuropathic pain \\ Molecular Signaling
}

\section{Introduction}

The spinal cord injury (SCI) is temporary or permanent damage in the spinal cord that disturbs the motor, sensory or autonomic functions of spinal cord. One of the SCI symptoms is peripheral and central chronic neuropathic pain (Ji et al., 2009). That is associated with excessive

\footnotetext{
* Corresponding author: Masoumeh Jorjani, msjorjani@sbmu.ac.ir

Received 11 August 2020; Revised from 17 March 2021; Accepted 6 April 2021
}

Citation: Alimoradian A, Abbaszadeh F, Jorjani M, Sadegh M. The behavioral and molecular effects of estradiol and progesterone on a rat model of spinothalamic tract lesion. Physiology and Pharmacology 2021; 25: 314-327. http://dx.doi.org/10.52547/phypha.25.4.9 
pain to painful stimuli (hyperalgesia) or spontaneous pain to stimuli, which are not normally perceived as painful (allodynia) (Chen and Pan, 2002). After the SCI and in the secondary phase, intense microglial activation and inflammatory mediators plays a major role in the creation and spread of long-lasting neuropathic pain ( Ji et al., 2009). Several events has been observed during the secondary damage like the enhancement of nitric oxide synthesis, oxidative stress, local inflammation, blood-brain barrier dysfunction, neuronal necrosis and apoptosis, scar and cyst formation, demyelination and disruption of the neuronal pathways. Therefore, it has been supposed the interventions with neuroprotective aims might be able to mitigate the inflammation and pathological consequences of secondary injury (Onose et al., 2009).

One of the principal signaling mechanisms involved in the secondary phase of SCI and hyperalgesia is the mitogen-activated protein kinases (MAPK) pathway (Ji et al., 2009; Ray et al., 2011). The MAP kinase pathway is important for the intracellular signal transduction during cellular response to diverse physiologic and pathologic conditions like the axon growth, microtubule and neurofilament phosphorylation, inflammation and pain (Kawasaki et al., 2006; Zhang et al., 2013). Three main members of MAPK signaling pathway including extracellular signal-regulated kinase (ERK), c-Jun N-terminal kinase (JNK) and p38 that play role in the molecular signaling for hyperalgesia after neuronal injury (Ji et al., 2009; Ray et al., 2011). Since MAP kinase contributes to hyperalgesia and neuronal inflammations, then the changes of biomarkers related to MAP kinase family after the SCI were considered in the present study.

Several therapeutic interventions like methylprednisolone, non-steroidal anti-inflammatory drugs and opioids have been used to reduce the pathologic processes of SCI. Most of them have limited and controversial efficacy (Sezer et al., 2015). Therefore, new approaches targeting secondary injury pathways are supposed to be more effective. One of these therapeutic perspectives is neurosteroids (di Michele et al., 2000). It has been shown that the neurosteroids produce in the dorsal root ganglia through a neuron-glia cross talk mechanism (Schaeffer et al., 2010). Their synthesis is increased after brain and spinal cord injury (di Michele et al., 2000; Coronel et al., 2016a). It has been reported that the neurosteroids can mitigate the SCI-induced neuropathic pain through effects on opioid systems in the CNS (Coronel et al., 2016a). They promote the remyelination and regeneration of injured nerves (Schaeffer et al., 2010). The different aspects of the neuroprotection by progesterone (Prog) have been observed (De Nicola et al., 2013; Ghorbanpoor et al., 2014). There are also many reports of the neuroprotective effects of the estradiol (Est) (Samantaray et al., 2011; Saghaei et al., 2013). Est can induce anti-inflammatory action via the estrogen receptor-dependent activation of MAPK (Garcia-Ovejero et al., 2005).

Given the importance of the inflammatory processes in the neural injury and neuropathic pain (Coronel et al., 2016b) changes in the related biomarkers have been investigated. Some of these markers are those linked to MAP kinases family or the markers of astrocytes and microglia activation like glial fibrillary acidic protein (GFAP) and ionized calcium-binding adaptor molecule 1 (Iba1). Therefore, in the present study, the effects of several doses of Est and Prog were studied during the unilateral lesion on the spinothalamic tract (STT).

\section{Material and methods}

\section{Animals}

Adult male Wistar rats ( $\mathrm{n}=77$; weighting 250-300g) were housed on a $12 \mathrm{~h}$ light/dark cycle and received food and water ad libitum. All experimental procedures were approved by the ethical committee of the Neuroscience Research Center of Shahid Beheshti University of Medical Sciences (ID: IR.SBMU.PHNS.REC.1394.57). All efforts were made to lower the animals suffering.

\section{Spinal lesion}

Rats were anesthetized using ketamine/xylazine $(60 / 20 \mathrm{mg} / \mathrm{kg}$, IP). The lesion was induced according to the method accomplished formerly (Saghaei et al., 2013) with a little modification in the protocol. Briefly, a dorsal laminectomy was performed at the right STT of spinal segments T8-T9. After exposure of the spinal cord, the dura was incised by a fine rongeur. Then, a tungsten electrode $(5 \mu \mathrm{m}$ tip, $1 \mathrm{M} \Omega)$ connected lesion making device (UGO Basile, Model 53500; Italy) was targeted to the right STT, based on stereotaxic coordinates (laterality to midline: $0.5-0.7 \mathrm{~mm}$ and depth: $1.6-1.9 \mathrm{~mm}$ ). The unilateral lesion was made by a brief current pulse $(300 \mu \mathrm{A}, 90 \mathrm{~s})$ passed through 
the electrode. After surgery, all animals received $1 \mathrm{ml}$ of saline to balance electrolytes as well as penicillin $G$ intramuscularly to prevent infection. Besides, during the surgery and recovery from anesthesia, the rats were covered with warm sterilized towels.

\section{Experimental groups}

Rats were divided into 11 groups including seven rats/ group. The sham group was subjected to laminectomy without electrolytic lesions. Injury group was subjected to the electrical lesions. Control group was subjected to lesion and $0.5 \mathrm{ml}$ of sesame oil as a vehicle. Other experimental groups were received different doses of Est or Prog (Sigma-Aldrich, St. Louis, MO) (2, 4, 8 or $16 \mathrm{mg} / \mathrm{kg}$ ) as post-treatment. All compounds were administered by intraperitoneal injection at $30 \mathrm{~min}$ postinjury.

\section{Behavioral assessment}

To determine the effects of STT lesion over longer time after central pain syndrome, the behavioral assessments, including motor activity and nociceptive responses, were investigated in separate groups on pre, 14 and 28 days post-surgery in all groups. The central pain syndrome can happen on day 14 and day 28 after STT lesion (Saghaei et al., 2013). Each animal was used as its own control, then pre and post-lesion responses were measured. The assessments were done by a blinded observer to the surgery. Only animals that were in the normal range of behavioral tests or no observe paralysis on STT-injured were selected for experiments.

\section{Mechanical allodynia}

Mechanical allodynia was evaluated as originally described by Ren (1999). Mechanical hind paw thresholds were measured bilaterally using calibrated von Frey filaments (Stoelting, IL, U.S.A). The earlier studies demonstrated that the dorsal approach detected more reliably and consistently the pain threshold changes after using calibrated von Frey filaments. It could detect the real nociceptive threshold (Lewin et al., 1993; Ren, 1999; Lucas et al., 2011). Then, the filaments were applied in ascending order to the dorsal surface of the hind paw between the second and third toes of hind paw the ipsi- and contralateral sides of the STT injury. The paw withdrawal threshold was defined as the force at which the animal withdrew the paw to three of the five stimuli delivered.

\section{Open field test}

Motor activity was also assessed using a $60 \times 60 \mathrm{~cm}$ arena and a $40 \mathrm{~cm}$ height black wooden box. Animals were habituated in the room for $30 \mathrm{~min}$ and then put in one corner of the box and allowed to explore freely for $5 \mathrm{~min}$. For each trial, the open field box was thoroughly cleaned with $70 \%$ ethanol solution and afterward by a dry cloth. The experiments were conducted under artificial laboratory illumination (fluorescent lamps, above the level of a box). The sessions were recorded by a camera positioned right above the box hanging from the ceiling. Data were obtained using Ethovision software (version 7), a video tracking system for automation of behavioral experiments (Noldus Information Technology, Netherlands). During the $5 \mathrm{~min}$ trial, the behavior of each rat was recorded as distance and velocity moved by rats in the open field box.

\section{Western blot}

At the end of behavioral assessments, the animals were sacrificed. The western blotting was performed in T8-9 spinal segments at the lesion site based on the previous report (Saghaei et al., 2013; Masoudi et al., 2017). The spinal tissue was homogenized with lysis buffer (150mM NaCl, $0.25 \%$ sodium deoxycholate, $0.1 \%$ Triton X-100, 50mM Tris-HCL, 0.1\% SDS, 1mM EDTA and $1 \%$ protease inhibitor cocktail, phosphatase inhibitor cocktail) by a tissue homogenizer. The total protein extract was obtained by centrifugation for $45 \mathrm{~min}$ at $12,000 \mathrm{rpm}$ at $4^{\circ} \mathrm{C}$. The protein concentration was measured using Bradford assay. Then, the protein samples were loaded on SDS-polyacrylamide gel electrophoresis and transferred to polyvinylidene difluoride membrane. The membranes were blocked with 5 and $3 \% \mathrm{w} / \mathrm{v}$ nonfat dry milk (Amersham, Ecl AdvanceTM) for $75 \mathrm{~min}$. Then they were incubated at $4{ }^{\circ} \mathrm{C}$ overnight with anti-GFAP (mouse, 1: 1,000, Cell signaling Co.), anti-Ibal (Goat polyclonal, 1: 1,000, Abcam Co.), anti-phospho-p44/42 (Thr202/Tyr204), ERK1/2 (Rabbit, 1: 1,000, Cell signaling Co.), anti-phospho-SAPK/JNK (Thr183/Tyr185), p46 and p54 (Rabbit,1: 1,000, Cell signaling Co.), anti-phospho-p38 (Thr180/Tyr182) (Rabbit,1: 1,000, Cell signaling Co.) and anti- $\beta$ actin (mouse, 1: 10,000, Cell Signaling) antibodies. Afterward, the membranes were incubated at room temperature for $2 \mathrm{~h}$ with horseradish 
peroxidase- conjugated secondary antibody (1:3000 $\mathrm{v} / \mathrm{v}$, Cell Signaling). The membranes were reacted with the enhanced chemiluminescence kit and then were exposed to x-ray films. The intensities of specific bands were quantified using the software Image J. Finally, the intensity values of the following marker were evaluated relative to the $\beta$-actin band's intensity: GFAP, Iba1, phospho-p38 (p-p38), phospho-ERK1 (p-ERK1), phospho-ERK2 (p-ERK2), phospho-ERK (p-ERK1/2) or p-ERK1+ p-ERK2, phospho-JNKp46 (pJNKp46), phospho-JNKp54 (pJNKp54), phospho-JNK (pJNK) or $\mathrm{pJNKp} 46+\mathrm{pJNKp} 54$. The ratios were considered as the average density for each marker. $\beta$-actin was used as the loading control for western samples of different groups.

\section{Immunohistochemistry}

The behavioral and western blot results expressed that Est at doses of $8 \mathrm{mg} / \mathrm{kg}$ could show the most significant effects. Then, to confirm these results, the changes of GFAP and Ibal expression induced by Est at doses of $8 \mathrm{mg} / \mathrm{kg}$ were compared with that in the sham, injury and control groups by immunohistochemistry. GFAP and Ibal are considered as markers of astrocytes and microglia activities respectively. The method was performed based on the previous report with brief modifications (Saghaei et al., 2013). Rats were deeply anesthetized with ketamine (70mg/kg IP) and transcardially perfused with phosphate-buffered saline (PBS) followed by $4 \%$ paraformaldehyde in PBS. Next, the spinal cord segments (T8-9) were removed and post-fixed in the same fixative overnight. After tissue dehydration/rehydration, spinal cord segments were embedded in paraffin. To immunohistochemical staining with antibodies, the 5- $\mu \mathrm{m}$ serial sections were provided by a microtome. The sections were deparaffinized in xylene, rehydrated through descending graded of ethanol. Antigen retrieval was carried out by microwaving in $10 \mathrm{mM}$ citrate buffer $(\mathrm{pH}=6)$ for $20 \mathrm{~min}$ to inactive endogenous peroxidase. The sections were immersed in 3\% hydrogen peroxide in $100 \%$ methanol for $15 \mathrm{~min}$. After several washings in PBS, the sections were incubated with $10 \%$ normal goat serum for $30 \mathrm{~min}$ at room temperature and then incubated overnight in humidified chambers at $4^{\circ} \mathrm{C}$ with primary antibody anti-GFAP (mouse, 1: 1,000, Cell signaling Co.) and anti-Iba1 (Goat polyclonal, 1: 1,000, Abcam Co.). Afterward, the sections were rinsed in PBS and incubated with a secondary antibody (EnVision+ Dual
Link System-HRP). The color reaction was developed by adding a chromogen, 3,3' -Diaminobenzidine tetrahydrochloride. Light counterstaining was carried out with hematoxylin. After dehydration by ascending graded alcohol and deparaffinization in xylene, the sections were coverslipped with Permount mounting medium (Entellan ${ }^{\circledR}$, MERK). The slides were visualized and images captured by a light microscope (Nikon, Japan) equipped with an optical camera. The cell count and density of GFAP and Ibal immunostaining was assessed semiquantitatively in 3 zones of the captured images separately and total zones by Image $\mathrm{J}$ software (NIH) (20× magnification).

\section{Histology}

The serial sections were prepared like just before immunohistochemistry staining. To verify the localization and extent of the lesion, the photo of each slide was taken by a microscope (Nikon). Data related to the rats with lesions outside of the spinal regions of interest were excluded. Figure 1 shows a representative histology section of the lesion site in the STT.

\section{Statistical analysis}

GraphPad Prism 5.0 (GraphPad Software, San Diego, California, USA) was used for statistical analysis and comparisons. The normal distribution of data was assessed by the "D'Agostino and Pearson omnibus normality test". Then the data were analyzed by one-way ANOVA followed by Tukey's multiple comparison test as post hoc. Data for each group during different days were also analyzed with repeated-measures ANOVA. A value of $P<0.05$ was considered statistically significant. All data are expressed as mean \pm SEM.

\section{Results}

\section{Mechanical allodynia}

Tables 1 and 2 show the effects of different doses of Est and Prog on the mechanical allodynia threshold. The pain level (gm) was shown in three steps, pre-surgery, 14 and 28th day after surgery on the contra and ipsilateral sides of the STT injury. There were no significant differences in the basal level of pain in all groups on the pre-surgery day. Following spinal cord injury, there was a significant decrease in the mechanical allodynia threshold in the groups that received Est compared to the injury or control groups. Treatment with Est at doses 
TABLE 1: the effects of different doses of estradiol (Est) and progesterone (Prog) on pain threshold (gm) of the hind paws, contralateral to the spinothalamic tract electrolytic lesion, during 0 (before surgery), 14 and 28 days post-surgery. As data show Est at doses 4, 8 and 16 significantly increase the pain threshold while Prog had no significant effect. Data are presented as mean \pm SEM. "Significant to control, ${ }^{*} P<0.05 ;{ }^{* *} P<0.01 ; \mathrm{n}=7$ for all groups.

\begin{tabular}{|c|c|c|c|}
\hline \multicolumn{4}{|c|}{ Contralateral } \\
\hline & Day 0 & $14^{\text {th }}$ day & $28^{\text {th }}$ day \\
\hline Sham laminectomy & $30.38 \pm 8.72$ & $46.50 \pm 6.044$ & $42.25 \pm 6.181$ \\
\hline Injury & $24.38 \pm 7.817$ & $16.25 \pm 3.075$ & $32 \pm 8.619$ \\
\hline Control & $42.75 \pm 7.27$ & $11.13 \pm 1.231$ & $35.13 \pm 11.78$ \\
\hline Est-2mg & $23.38 \pm 5.571$ & $32.5 \pm 6.31$ & $57.25 \pm 10.86$ \\
\hline Est-4mg & $27.63 \pm 7.457$ & $59.5 \pm 11.06^{* *}$ & $55.88 \pm 11.5$ \\
\hline Est-8mg & $30.25 \pm 6.92$ & $62.5 \pm 8.015^{* *}$ & $84.38 \pm 11.08^{*}$ \\
\hline Est-16mg & $38.29 \pm 7.174$ & $44.14 \pm 8.481$ & $81.43 \pm 13.17^{*}$ \\
\hline Prog-2mg & $28.13 \pm 7.274$ & $27.25 \pm 7.556$ & $57.25 \pm 10.86$ \\
\hline Prog-4mg & $20.13 \pm 5.992$ & $34.75 \pm 7.889$ & $52.25 \pm 13.01$ \\
\hline Prog-8mg & $33.38 \pm 8.091$ & $21.75 \pm 6.067$ & $59.25 \pm 11.22$ \\
\hline Prog-16mg & $25.38 \pm 5.791$ & $34.5 \pm 6.299$ & $69.38 \pm 10.46$ \\
\hline
\end{tabular}

TABLE 2: The effects of different doses of estradiol (Est) and progesterone (Prog) on pain threshold (gm) of the hind paws, ipsilateral to the spinothalamic tract electrolytic lesion, during 0 (before surgery), 14 and 28 days post-surgery. As data show Est at dose 8 significantly increase the pain threshold while Prog had no significant effect. Data are presented as mean \pm SEM. "Significant to control, ${ }^{*} P<0.05 ;{ }^{* *} P<0.01 ; \mathrm{n}=7$ for all groups.

\begin{tabular}{|c|c|c|c|}
\hline \multicolumn{4}{|c|}{ Ipsilateral } \\
\hline & Day 0 & $14^{\text {th }}$ day & $28^{\text {th }}$ day \\
\hline Sham laminectomy & $33.75 \pm 8.252$ & $41.38 \pm 14.08$ & $52.38 \pm 9.059$ \\
\hline Injury & $30.25 \pm 7.004$ & $31 \pm 15.87$ & $36.25 \pm 7.032$ \\
\hline Control & $17.88 \pm 3.176$ & $22.25 \pm 6.731$ & $43.25 \pm 11.23$ \\
\hline Est-2mg & $22.75 \pm 5.688$ & $41.75 \pm 10.4$ & $55.25 \pm 11.83$ \\
\hline Est-4mg & $29.5 \pm 7.046$ & $76.5 \pm 18.58$ & $63.75 \pm 12.49$ \\
\hline Est-8mg & $24.38 \pm 5.732$ & $130 \pm 19.64^{* * *}$ & $80.57 \pm 7.581^{*}$ \\
\hline Est-16mg & $37.29 \pm 6.516$ & $47.29 \pm 12.1$ & $75.00 \pm 7.319$ \\
\hline Prog-2mg & $16.25 \pm 3.075$ & $40.25 \pm 14.42$ & $42.38 \pm 10.15$ \\
\hline Prog-4mg & $20.88 \pm 6.11$ & $42.38 \pm 11.57$ & $51.63 \pm 12.05$ \\
\hline Prog-8mg & $34.38 \pm 8.022$ & $23.38 \pm 5.997$ & $53.88 \pm 12.38$ \\
\hline Prog-16mg & $16.63 \pm 2.982$ & $57.25 \pm 12.21$ & $65 \pm 5$ \\
\hline
\end{tabular}

4 and $8 \mathrm{mg} / \mathrm{kg}$ increased the pain threshold on day 14 and at doses 8 and 16 on day 28 after surgery on the contralateral side of the STT. The same increase was observed at the threshold of pain at a dose of $8 \mathrm{mg} / \mathrm{kg}$ of Est on both days 14 and 28 on the ipsilateral sides of the STT. While Prog had no significant effect on the threshold of pain compared to the injury or control groups.

The pharmacological potencies of two steroids were compared by effective dose $50 \%$ (ED50) defined as the logarithm (base 10) of the steroid dose $(\mathrm{mg} / \mathrm{kg}$ ) required to elicit $50 \%$ of the maximal effect. The comparison of ED50 for two steroids revealed that Est was more potent than Prog during the $14^{\text {th }}$ and 28 th days after surgery on both of paws. The ED50 on the $14^{\text {th }}$ day after surgery was as follows: Est (5.444), Prog (19.7) for the contralateral; Est (1.544) and Prog (10.08) for the ipsilateral sides. Also, the value of ED50 on the $28^{\text {th }}$ day was Est (2.09) and Prog (3.38) for the contralateral; Est (2.144) and Prog (4.774) for the ipsilateral sides.

Considering more effect of Est in comparison with control and injury groups and the less ED50, it seems Est has more efficient for the increase of pain threshold after spinal injury compared to Prog.

\section{Motor activity}

Tables 3 and 4 show the effects of different doses of Est and Prog on the moved distance $(\mathrm{cm})$ and run 
TABLE 3: The effects of different doses of estradiol (Est) and progesterone (Prog) on the moved distance (cm) during $5 \mathrm{~min}$ in the open-field test on day 0 (before surgery), 14 and 28 post-surgery. As data show Est at dose $8 \mathrm{mg} / \mathrm{kg}$ significantly increase the distance while Prog had no significant effect. Data are presented as mean \pm SEM. ${ }^{*}$ Significant to control, ${ }^{*} P<0.05$; $\mathrm{n}=7$ for all groups.

\begin{tabular}{|c|c|c|c|}
\hline \multicolumn{4}{|c|}{ Distance $(\mathrm{cm})$} \\
\hline & Day 0 & $14^{\text {th }}$ day & $28^{\text {th }}$ day \\
\hline Sham laminectomy & $1836 \pm 132.1$ & $1621 \pm 87.77$ & $1589 \pm 97.61$ \\
\hline Injury & $1821 \pm 95.7$ & $1424 \pm 115.4$ & $1652 \pm 315.6$ \\
\hline Control & $1965 \pm 88.19$ & $1501 \pm 152.2$ & $1795 \pm 145.9$ \\
\hline Est-2mg & $2025 \pm 128.9$ & $1655 \pm 150.9$ & $1542 \pm 142.2$ \\
\hline Est-4mg & $1814 \pm 88.94$ & $1860 \pm 105$ & $1533 \pm 241.9$ \\
\hline Est-8mg & $1811 \pm 133.7$ & $1962 \pm 90.86^{*}$ & $1553 \pm 157$ \\
\hline Est-16mg & $1896 \pm 120.2$ & $1735 \pm 102.6$ & $1915 \pm 200.8$ \\
\hline Prog-2mg & $1944 \pm 78.86$ & $1584 \pm 81.64$ & $2012 \pm 218.2$ \\
\hline Prog-4mg & $2036 \pm 105.8$ & $1530 \pm 102.7$ & $1691 \pm 200.7$ \\
\hline Prog-8mg & $2074 \pm 225$ & $1732 \pm 69.17$ & $1742 \pm 186.1$ \\
\hline Prog-16mg & $2021 \pm 152.8$ & $1603 \pm 66.38$ & $1815 \pm 352.2$ \\
\hline
\end{tabular}

TABLE 4: The effects of different doses of estradiol (Est) and progesterone (Prog) on the run velocity $(\mathrm{cm} / \mathrm{s})$ during $5 \mathrm{~min}$ in the open-field test on day 0 (before surgery), 14 and 28 post-surgery. No significant effect was detected from Est or Prog. Data are presented as mean \pm SEM. $n=7$ for all groups.

\begin{tabular}{|c|c|c|c|}
\hline \multicolumn{4}{|c|}{ Velocity $(\mathrm{cm} / \mathrm{s})$} \\
\hline & Day 0 & $14^{\text {th }}$ day & $28^{\text {th }}$ day \\
\hline Sham laminectomy & $7.742 \pm 0.856$ & $5.243 \pm 0.5011$ & $10.19 \pm 0.8692$ \\
\hline Injury & $6.638 \pm 0.8562$ & $4.633 \pm 0.5489$ & $9.633 \pm 0.496$ \\
\hline Control & $6.884 \pm 0.601$ & $5.488 \pm 0.3407$ & $12.05 \pm 0.2892$ \\
\hline Est-2mg & $7.937 \pm 0.6586$ & $7.101 \pm 0.3369$ & $9.223 \pm 1.046$ \\
\hline Est-4mg & $8.148 \pm 0.6816$ & $5.936 \pm 0.2221$ & $9.268 \pm 0.8106$ \\
\hline Est-8mg & $7.382 \pm 0.2675$ & $6.129 \pm 0.2925$ & $10.892 \pm 0.413$ \\
\hline Est-16mg & $7.344 \pm 0.5493$ & $7.258 \pm 0.2007$ & $10.41 \pm 1.004$ \\
\hline Prog-2mg & $7.579 \pm 0.5246$ & $5.965 \pm 0.373$ & $12.97 \pm 2.316$ \\
\hline Prog-4mg & $8.054 \pm 0.6513$ & $5.732 \pm 0.2621$ & $6.219 \pm 0.7376$ \\
\hline Prog-8mg & $7.811 \pm 0.66$ & $6.189 \pm 0.2268$ & $16.19 \pm 2.172$ \\
\hline Prog-16mg & $7.484 \pm 0.7682$ & $5.735 \pm 0.314$ & $8.513 \pm 1.327$ \\
\hline
\end{tabular}

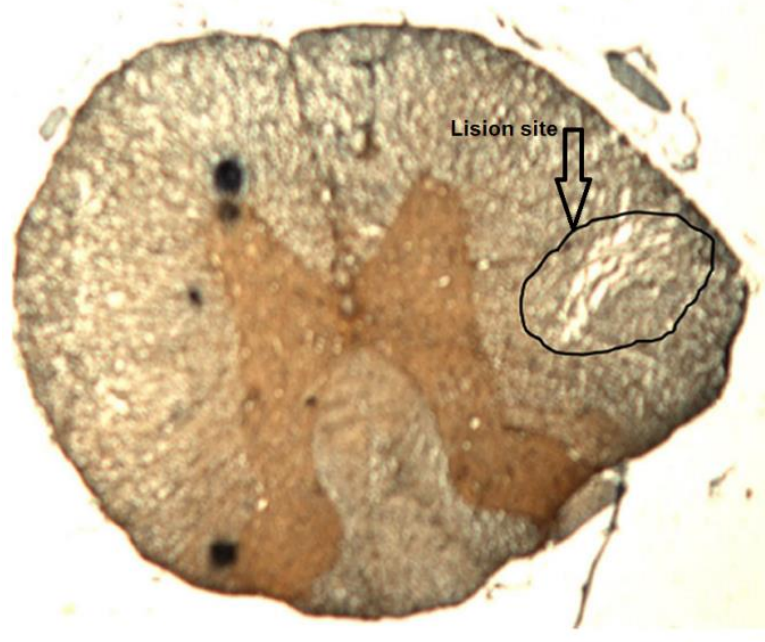

FIGURE 1. Histological verification of the lesion location. The area pointed by the arrow is the location of the lesion electrode. 


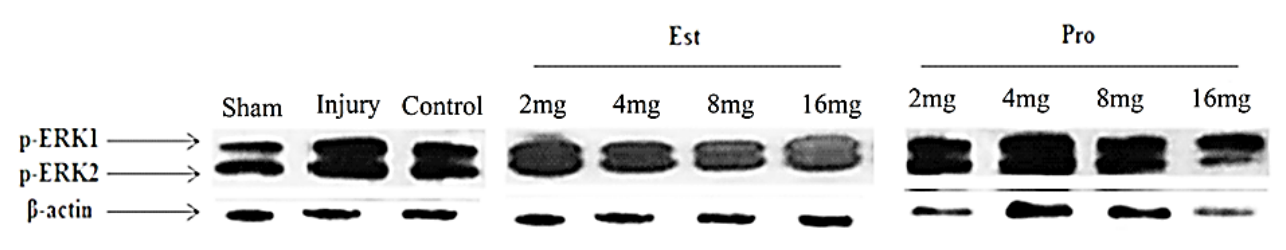

A)

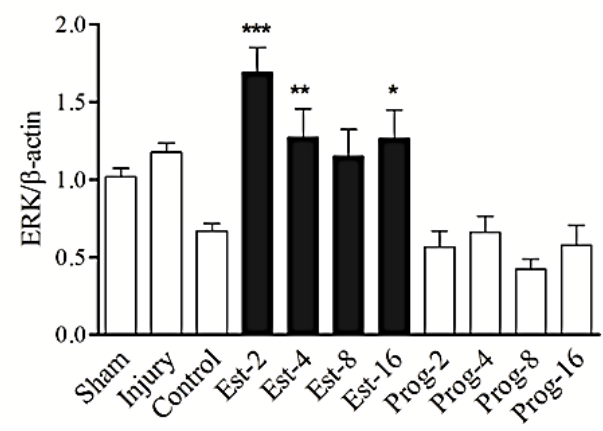

B)

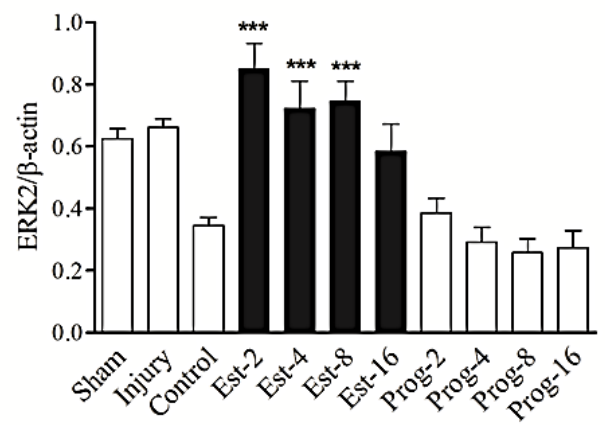

FIGURE 2. The effects of different doses of Est and Prog on the activation of p-ERK after spinothalamic tract injury on the 28th day. All doses of Est significantly increased the p-ERK level while Prog had no significant effects. Data are presented as mean \pm SEM for the average relative density of phospho-p44/42 mitogen-activated protein kinases (MAPKs) to $\beta$-actin. "Significant to control, ${ }^{*} P<0.05 ;{ }^{* * *} P<0.01 ;{ }^{* * *} P<0.001 ; \mathrm{n}=7$ for all groups.
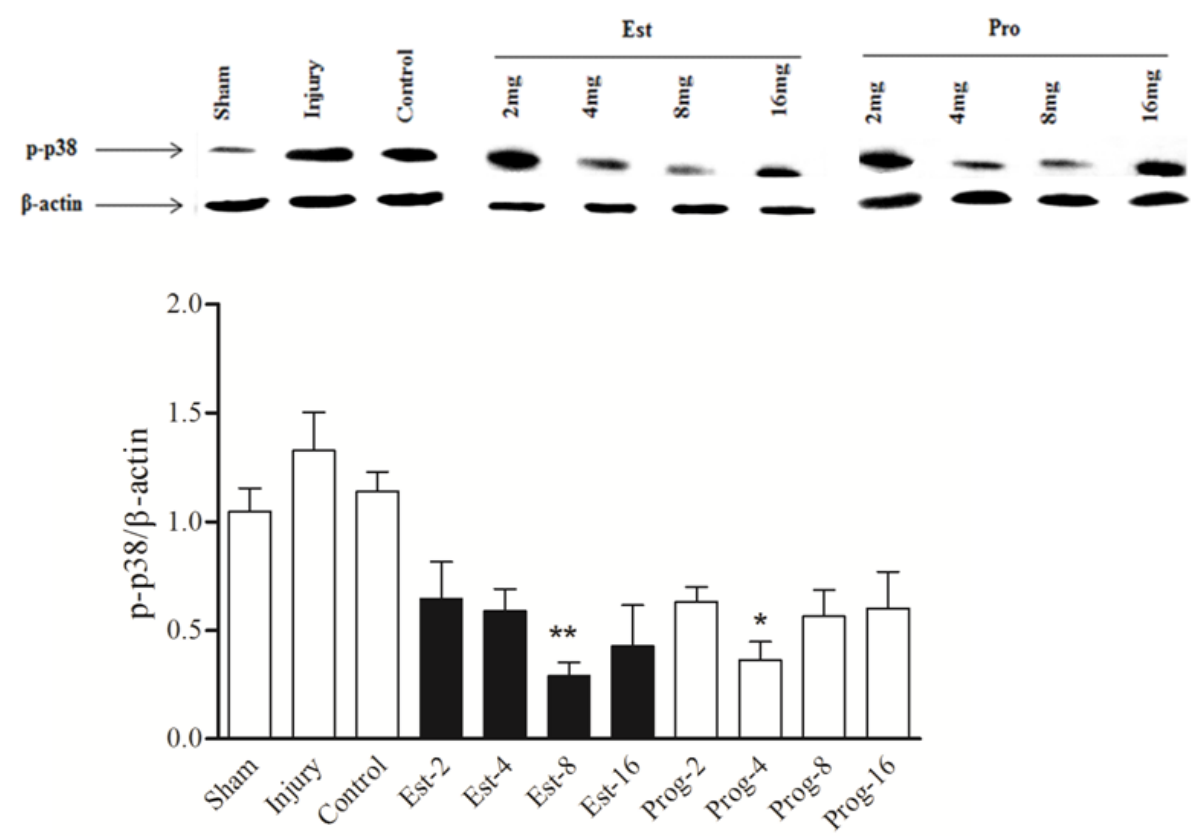

FIGURE 3. The effects of different doses of Est and Prog on the activation of p-P38 in rats after spinothalamic tract injury on the $28^{\text {th }}$ day. Both Est $8 \mathrm{mg} / \mathrm{kg}$ and Prog $4 \mathrm{mg} / \mathrm{kg}$ significantly decreased the p-P38 in comparison with control group. Data are presented as mean \pm SEM for the average relative density of phospho-P38 mitogen-activated protein kinases (p-p38) (MAPKs) to $\beta$-actin. ${ }^{*}$ Significant to control, ${ }^{*} P<0.05$; ${ }^{* *} P<0.01 ;{ }^{* * *} P<0.001 ; \mathrm{n}=7$ for all groups.

velocity $(\mathrm{cm} / \mathrm{s})$ respectively. The motor parameters were shown in three steps, pre-surgery, 14 and $28^{\text {th }}$ day after surgery. Est increased the distance and velocity higher than did injury group, respectively in doses of 8 and $16 \mathrm{mg} / \mathrm{kg}$ on the $14^{\text {th }}$ day after surgery. Two other doses of Est didn't have any significant effect. The treatment with different doses of Prog had no significant effect on the improvement of motor function in days after surgery.

\section{Western blot analysis}

The expression of the following parameters was investigated as astroglia activity markers: GFAP, Iba1, and MAP kinase family like p-p38, p-ERK, and pJNK. The intensity of marker bands relative to $\beta$-actin was 

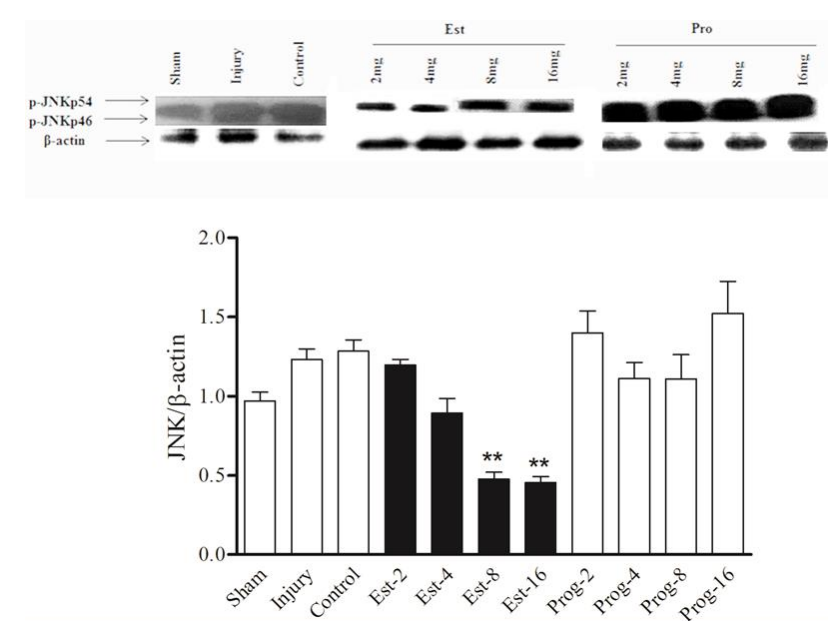

FIGURE 4. The effects of different doses of Est and Prog on the activation of p-JNK in rats after spinothalamic tract injury on the 28th day. Est at doses 8 and $16 \mathrm{mg} / \mathrm{kg}$ significantly decreased the $\mathrm{p}-\mathrm{JNK}$ level while Prog had no significant effects. Data are presented as mean \pm SEM for the average relative density of JNK to $\beta$-actin. "Significant to control, ${ }^{*} P<0.05 ;{ }^{* *} P<0.01 ;{ }^{* * *} P<0.001 ; \mathrm{n}=7$ for all groups.
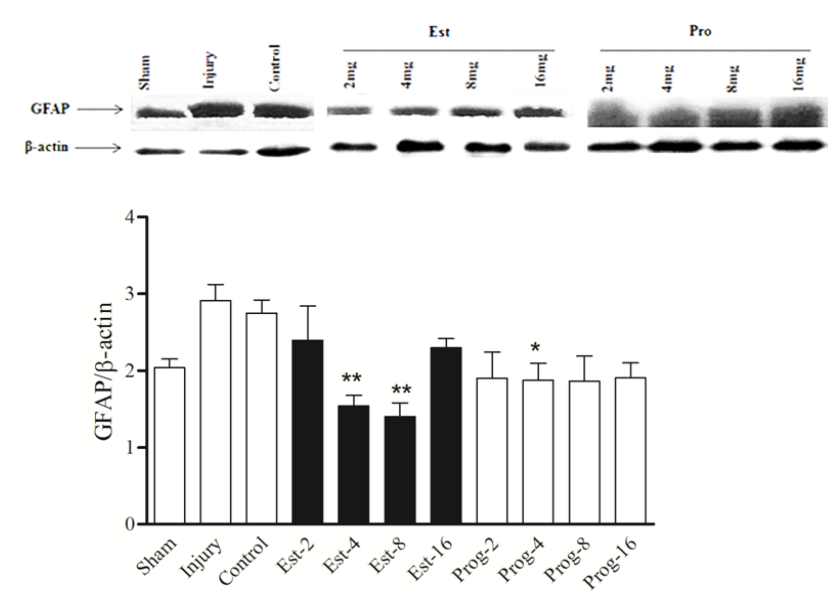

defined as the average density. Quantified densitometric data showed that Est and Prog had different effects on 3 subtypes of the MAP kinase family. Following spinal cord injury, the level of p-ERK expression was significantly reduced in the injury or control groups. Est at all doses increased the expression of p-ERK in comparison with the control $(P<0.01$, Figure 2$)$; while no significant effects of Prog were observed.

After spinal cord injury, the expression level of p-p38 and pJNK increased on day 28 in the injury and control groups. Treatment with Est $8 \mathrm{mg} / \mathrm{kg}$ and Prog $4 \mathrm{mg} / \mathrm{kg}$ significantly reduced the expression of p-p38 compared to the control and injury groups $(P<0.01$, Figure 3$)$. In addition, treatment with Est $8 \mathrm{mg} / \mathrm{kg}$ and $16 \mathrm{mg} / \mathrm{kg}$ significantly reduced the expression of $\mathrm{pJNK}$ compared to the control and injury groups $(P<0.01$, Figure 4$)$.
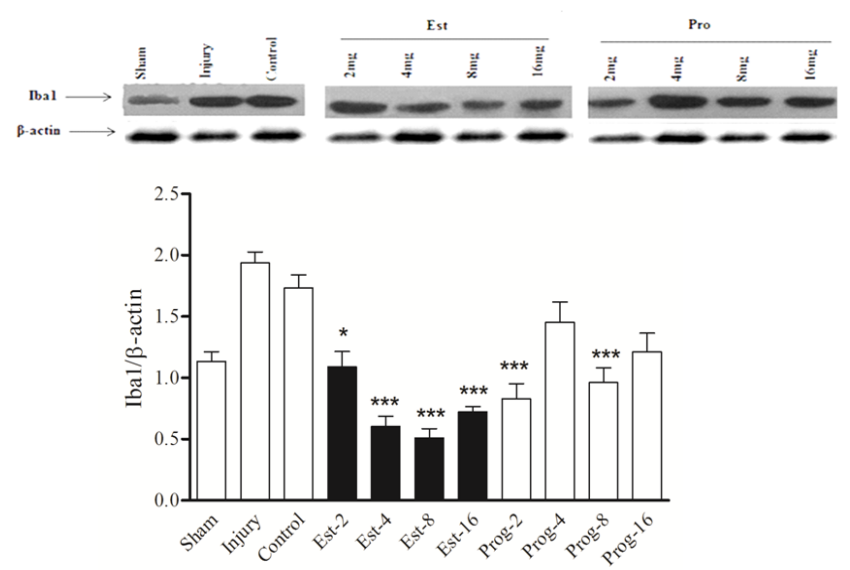

FIGURE 5. The effects of different doses of Est and Prog on the activation of Ibal in rats after spinothalamic tract injury. on the 28th day. Both Est and Prog significantly decreased the Ibal level in compare with control. Data are presented as mean \pm SEM for the average relative density of Ibalto $\beta$-actin. " Significant to control, ${ }^{*} P<0.05$; ${ }^{* *} P<0.01 ;{ }^{* * *} P<0.001 ; \mathrm{n}=7$ for all groups.
FIGURE 6. The effects of different doses of Est and Prog on the activation of GFAP in rats after spinothalamic tract injury on the 28th day. Both Est at doses 4 and $8 \mathrm{mg} / \mathrm{kg}$ and Prog at dose $4 \mathrm{mg} / \mathrm{kg}$ significantly decreased the GFAP level. Data are presented as mean \pm SEM for the average relative density of GFAP to $\beta$-actin. *Significant to control, ${ }^{*} P<0.05 ; * * P<0.01 ; * * * P<0.001 ; \mathrm{n}=7$ for all groups.

Following spinal cord injury, the expression of Iba1 and GFAP was increased in the injury and control groups. All different doses of Est and doses of 2 and $8 \mathrm{mg} / \mathrm{kg}$ OD Prog were able to significantly reduced the expression level of the Iba1 marker $(P<0.001$, Figure 5). Also, Est in doses 4 and $8 \mathrm{mg} / \mathrm{kg}$ significantly reduced the expression level of the GFAP $(P<0.01$, Figure 6$)$.

\section{Immunohistochemistry}

Considering that Est at the dose of $8 \mathrm{mg} / \mathrm{kg}$ was more effective than the other doses used in reducing the pain threshold and molecular outcomes, it was investigated by immunohistochemistry. The comparison of the immunostained sections showed a significantly less count in the expression of GFAP (Figures 7A and B) and Iba1 (Figures 7C and D) in Est $8 \mathrm{mg} / \mathrm{kg}$ compared to the 

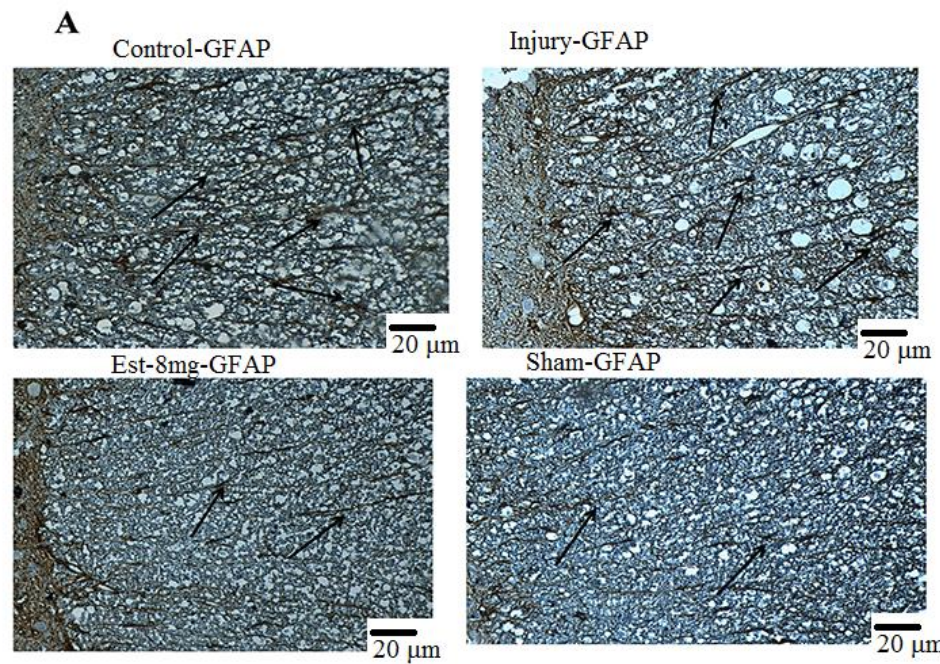

\section{C}
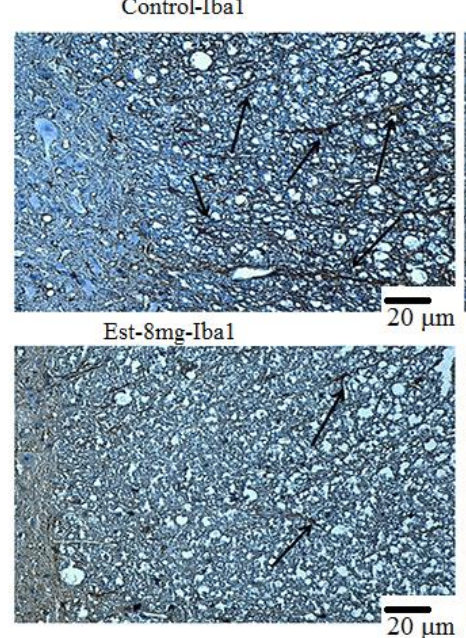

Injury-Iba1



B

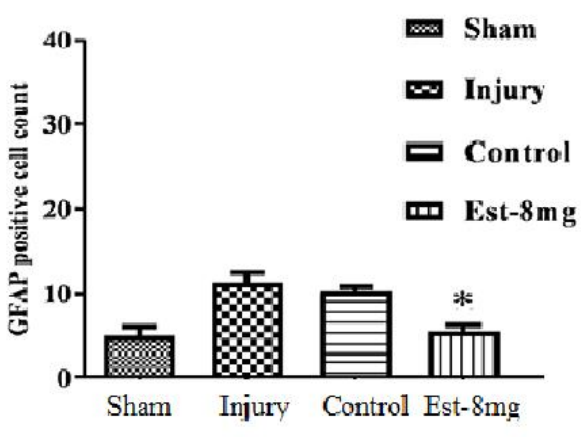

D

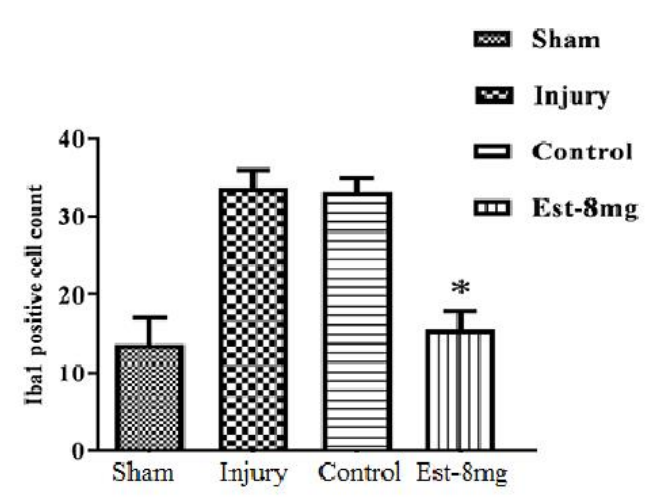

FIGURE 7. Expression of GFAP and Iba1 in spinal cord sections at T8-10 level after spinothalamic tract injury on the 28th day. the effect of Est $8 \mathrm{mg} / \mathrm{kg}$ on the GFAP positive cell count (A \& B); the effect of Est $8 \mathrm{mg} / \mathrm{kg}$ on the Iba1 positive cell count (C \& D). Est 8mg/kg significantly

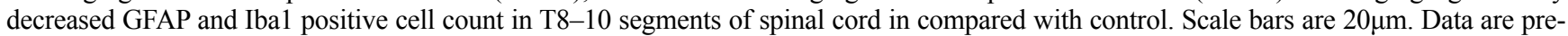
sented as mean \pm SEM. *Significant to group; ${ }^{*} P<0.05 ; \mathrm{n}=7$ for all groups.

control group.

\section{Discussion}

In the present study, the effects of different doses of Est and Prog on SCI were studied. Two steroids were injected intraperitoneally into rats following an electrolytic lesion on the STT. Est showed higher efficacy to reduce the threshold of pain and neuronal survival, especially in doses of 4 and $8 \mathrm{mg} / \mathrm{kg}$ compared to the control group and Prog. Thus, Est mitigated the inflammatory markers of MAP-kinases subtypes like p-38 and JNK as well as astroglial activity such as Iba1 and GFAP. Also, Est increased the ERK expression while Prog show no effect, which confirmed Est positive impact on neuronal survival. Also, in this study, the effects of Est and Prog on biomarkers were evaluated on the $28^{\text {th }}$ day after surgery.

Est mediates the neuroprotection signaling through distinct pathways like the nuclear estrogen receptor regulation of transcription, non-genomic signaling via the MAP or phosphatidylinositol-3 kinase (PI3-K) with its effector down-stream a serine/threonine kinase and finally via non-receptor pathway that in trapped free radical as anti-oxidant (Honda et al., 2000; Ivanova et al., 2002). Estrogen interacts directly with the MAP kinase pathway that activates the signaling molecules such as Ras sequentially. Afterward, a variety of transcription factors involved in neuronal survival will activate 
(Morissette et al., 2008). This interaction is especially important for neuroprotection after neuronal toxicity events (Singer et al., 1999). So that, Est decreases intracellular $\mathrm{Ca}$, activates ERK1/2 in midbrain astroglia (Ivanova et al., 2001; Ray et al., 2011), affects the PI3-K/Akt signal transduction pathway (Ivanova et al., 2002; Wilson et al., 2002). Thereby Est promotes neuronal survival and differentiation while protects against excitotoxicity and apoptosis. Est triggers the neurochemical changes in GABAergic neurons and opioid receptors in the spinal cord and the periaqueductal gray resulting in reduced inflammatory mediators such as PGE2, metalloproteinase-9 (Garcia-Ovejero et al., 2005) and pain sensitivity (Saghaei et al., 2013). That may be relevant for explaining why Est can control pain more efficient than that of Prog at this work.

Est $(4 \mathrm{mg} / \mathrm{kg})$ as acute treatment can attenuate the edema, inflammation and neutrophil infiltration after SCI. It inhibits the protein complex by which controls cytokine production like NF- $\mathrm{BB}$ (nuclear factor kappalight-chain-enhancer of activated B cells). Est relieves the axonal degeneration and myelin loss as well as the activity of calpain and caspase- 3 in penumbra segments of SCI lesion (Sribnick et al., 2010; Tohda and Kuboyama, 2011). The induction of anti-inflammatory effects of Est on the supraphysiologic dose is consistent with our observation in which, high dose of $8 \mathrm{mg} / \mathrm{kg}$ Est showed more efficient for the analgesic and the inhibition of the cells involving in inflammation like the astrocyte and microglia. Est mitigated the biomarkers of astrocyte activity like GFAP and JNK as well as the biomarkers of the microglia activity like p-p38 or Iba1. The GFAP and JNK are considered as the astrocyte activity markers (Sanna et al., 2015) while the p-p38 or Ibal for the microglia activity markers (Patel et al., 2016). The activation of JNK and p38 MAPK take part in neuronal death (Singer et al., 1999) and the inhibition of these subtypes of MAPK by Est may be critical to its effect.

The hyperactivity of spinal microglia and the STT neurons is important in the pathogenesis of neuropathic pain (Tozaki-Saitoh et al., 2008). Est inhibits inducible nitric oxide synthase and cyclooxygenase-2, by which can reverse the microglial excitation and lessen the STT damage-induced pain (Samantaray et al., 2010; Sribnick et al., 2010; Samantaray et al., 2011). Also, the overexpression of G-protein coupled receptors of estrogen (alpha and beta subtypes), glutathione and superoxide dismutase have been observed in the reactive astrocytes of the STT lesions, thereby Est could put on its antioxidant mechanism (Onose et al., 2009; Mosquera et al., 2014). Furthermore, Est modulates L-type Ca2+ channels and the $\mathrm{Na}+-\mathrm{Ca} 2+$-exchanger leading to the alleviation of $\mathrm{Ca} 2+$ influx (Samantaray et al., 2011), scavenging of free radical intracellular like $[\mathrm{Ca} 2+]$ (Das et al., 2011) and improving mitochondrial function (Sribnick et al., 2005).

Nevertheless, neuroprotective effects of Est could be mediated through the mitigation of apoptosis pathways (Elkabes and Nicot, 2014). Est modifies the phosphorylation of cAMP response element-binding protein at Ser (Bonin et al., 2014). The down-stream of change may be the attenuation of the proapoptotic markers like Bad and Bax (Wilson et al., 2002; Morissette et al., 2008; Samantaray et al., 2010; Samantaray et al., 2011; Bonin et al., 2014). Meanwhile, Est amplifies the anti-apoptotic factors such as Bcl-2 (Honda et al., 2001) or a serine/threonine-specific protein kinase as protein kinase B or Akt which have a great influence on cellular survival pathways (Ivanova et al., 2002) and promotion of the growth factors (Bramanti et al., 2015). Our observation showed that Est and Prog could decrease p-p38 significantly compared with injured and control groups. The activation of p38 in microglia results in the increase of pro-inflammatory cytokines such as TNF $\alpha$, IL-1 $\beta$ and IL-6 (Taves et al., 2016). The rapid up-regulation of cytokines happens after an injury in the spinal tissue (Ritz and Hausmann, 2008) and neurosteroids can down-regulate them (Garcia-Ovejero et al., 2005; Ritz and Hausmann, 2008; Brotfain et al., 2016; Coronel et al., 2016b). This evidence explains the anti-inflammatory and analgesic effects of Est observed in the present study.

It was found in this study that Est was able to reduce JNK and p38 while raising ERK. Previous works have shown that the activation of ERK along with the inhibition of JNK and p38 MAPK is associated with cell survival (Singer et al., 1999). Therefore, the observed effects of Est on MAP kinase family in this work may explain a neuroprotective role for Est. All estrogen receptor subtypes have been found in oligodendrocytes (Lee et al., 2012; Elkabes and Nicot, 2014), but two subtypes of estrogen receptor, ER $\alpha$ and ER $\beta$ (Morissette et al., 2008) especially ER $\beta$ as the main isoform are 
expressed in oligodendrocytes of the spinal cord (Elkabes and Nicot, 2014). Est through these receptors can apply the neuroprotective effects. Down-stream of receptor binding leads to the activation of MAP kinase signal transduction (Singer et al., 1999), the inhibition of JNK (Lee et al., 2012) or activation of ERK1/2 in astrocytes (Ivanova et al., 2001). As a result, the sequential events like the phosphorylation of Ras, B-Raf take place by which mediate the neuronal survival while the modulation of the proteins influencing cell death (Wilson et al., 2002; Morissette et al., 2008; Bonin et al., 2014) or inflammation (Singer et al., 1999; GarciaOvejero et al., 2005). Moreover, the MAPK pathway interacts with the insulin-like growth factor-I receptor as a critical event for neuronal survival (Dhandapani and Brann, 2002; Morissette et al., 2008). Eventually, the phosphorylation of cytoskeletal proteins such as microtubule-associated proteins and neurofilaments will take place (Dominguez et al., 2004). That would explain the Est efficacy for neuronal plasticity in morphological changes related to the synaptogenesis, dendritic arborization and finally the neuronal survival and differentiation (Ivanova et al., 2002; Wilson et al., 2002; Garcia-Ovejero et al., 2005).

These conflicting effects of Prog on the improvement of spinal cord injuries have been reported earlier. Prog has a positive effect on different types of inflammatory biomarkers induced by microglial activation or myelin regeneration following SCI. However, it has not been able to relieve most issues of the neuronal damage after the spinal cord injury (Elkabes and Nicot, 2014). Prog inhibits the microglia activation (Garcia-Ovejero et al., 2014), which can justify the decrease in p38 or Iba1 as a measure of microglial proliferation (Taves et al., 2016) observed in the current study. Prog modulates glutamate release (Brotfain et al., 2016) and prevents the upregulation of glutamate receptors (Coronel et al., 2017). By inhibiting pro-inflammatory cytokine expression (Coronel et al., 2016a; Coronel et al., 2016b), Prog can reverse the neuropathic pain (Gonzalez and Coronel, 2016) after SCI or central injuries. However, we observed the contradictory results for Prog in the present work. It may be associated with the different conditions or doses used in the present study. As at least there is a report on the effects of Prog exacerbation in brain lesions in certain conditions (De Nicola et al., 2009) or lack of preventive effects in spinal cord injuries (Cavalcante et al., 2018).

\section{Conclusion}

Taken together, it seems that Est decreases the threshold of pain and the inflammatory markers during the secondary damage phase after SCI, while increases the survival factor more efficiently than Prog. Est applies the neuroprotective effects through distinct pathways like the modulation of astroglial activity or the MAP kinase pathway. Therefore, it can exert more efficient neuroprotection after SCI.

\section{Conflicts of interest}

The authors declare that there are no conflicts of interest.

\section{Acknowledgment}

This study was funded by the School of Medicine and Neurobiology Research Center of Shahid Beheshti University of Medical Sciences (Grant No 209).

\section{References}

Bonin RP, Bories C, De Koninck Y. A simplified up-down method (sudo) for measuring mechanical nociception in rodents using von frey filaments. Mol Pain 2014; 10: 26. https://doi.org/10.1186/1744-8069-10-26

Bramanti V, Grasso S, Tibullo D, Giallongo C, Raciti G, Viola $\mathrm{M}$, et al. Modulation of extracellular signal-related kinase, cyclin d1, glial fibrillary acidic protein, and vimentin expression in estradiol-pretreated astrocyte cultures treated with competence and progression growth factors. J Neurosci Res 2015; 93: 1378-87. https://doi.org/10.1002/ jnr.23606

Brotfain E, Gruenbaum SE, Boyko M, Kutz R, Zlotnik A, Klein M. Neuroprotection by estrogen and progesterone in traumatic brain injury and spinal cord injury. Curr Neuropharmacol 2016; 14: 641-53. https://doi.org/10.2174/15701 59X14666160309123554

Cavalcante LP, Ferreira SG, Pereira DR, Moraes SR, Simas R, Sannomiya P, et al. Acute administration of oestradiol or progesterone in a spinal cord ischaemia-reperfusion model in rats. Interact Cardiovasc Thorac Surg 2018; 26: 196-201. https://doi.org/10.1093/icvts/ivx314

Chen SR, Pan HL. Hypersensitivity of spinothalamic tract neurons associated with diabetic neuropathic pain in rats. J Neurophysiol 2002; 87: 2726-33. https://doi.org/10.1152/ jn.2002.87.6.2726 
Coronel MF, Labombarda F, Gonzalez SL. Neuroactive steroids, nociception and neuropathic pain: a flashback to go forward. Steroids 2016a; 110: 77-87. https://doi. org/10.1016/j.steroids.2016.04.005

Coronel MF, Raggio MC, Adler NS, De Nicola AF, Labombarda F, Gonzalez SL. Progesterone modulates pro-inflammatory cytokine expression profile after spinal cord injury: implications for neuropathic pain. J Neuroimmunol 2016b; 292: 85-92. https://doi.org/10.1016/j.jneuroim.2016.01.011

Coronel MF, Villar MJ, Brumovsky PR, Gonzalez SL. Spinal neuropeptide expression and neuropathic behavior in the acute and chronic phases after spinal cord injury: effects of progesterone administration. Peptides 2017; 88: 189-95. https://doi.org/10.1016/j.peptides.2017.01.001

Das A, Smith JA, Gibson C, Varma AK, Ray SK, Banik NL. Estrogen receptor agonists and estrogen attenuate tnf-alpha-induced apoptosis in vsc4.1 motoneurons. J Endocrinol 2011; 208: 171-82. https://doi.org/10.1677/JOE-100338

De Nicola AF, Coronel F, Garay LI, Gargiulo-Monachelli G, Gonzalez Deniselle MC, Gonzalez SL, et al. Therapeutic effects of progesterone in animal models of neurological disorders. CNS Neurol Disord Drug Targets 2013; 12: 1205-18.

De Nicola AF, Labombarda F, Gonzalez Deniselle MC, Gonzalez SL, Garay L, Meyer M, et al. Progesterone neuroprotection in traumatic cns injury and motoneuron degeneration. Front Neuroendocrinol 2009; 30: 173-87. https://doi. org/10.1016/j.yfrne.2009.03.001

Dhandapani KM, Brann DW. Protective effects of estrogen and selective estrogen receptor modulators in the brain. Biol Reprod 2002; 67: 1379-85. https://doi.org/10.1095/ biolreprod.102.003848

di Michele F, Lekieffre D, Pasini A, Bernardi G, Benavides J, Romeo E. Increased neurosteroids synthesis after brain and spinal cord injury in rats. Neurosci Lett 2000; 284: 65-8. https://doi.org/10.1016/S0304-3940(00)00965-4

Dominguez R, Jalali C, de Lacalle S. Morphological effects of estrogen on cholinergic neurons in vitro involves activation of extracellular signal-regulated kinases. J Neurosci 2004; 24: 982-90. https://doi.org/10.1523/JNEUROSCI.2586-03.2004

Elkabes S, Nicot AB. Sex steroids and neuroprotection in spinal cord injury: a review of preclinical investigations. Exp Neurol 2014; 259: 28-37. https://doi.org/10.1016/j.expneurol.2014.01.008
Garcia-Ovejero D, Azcoitia I, Doncarlos LL, Melcangi RC, Garcia-Segura LM. Glia-neuron crosstalk in the neuroprotective mechanisms of sex steroid hormones. Brain Res Brain Res Rev 2005; 48: 273-86. https://doi.org/10.1016/j. brainresrev.2004.12.018

Garcia-Ovejero D, Gonzalez S, Paniagua-Torija B, Lima A, Molina-Holgado E, De Nicola AF, et al. Progesterone reduces secondary damage, preserves white matter, and improves locomotor outcome after spinal cord contusion. J Neurotrauma 2014; 31: 857-71. https://doi.org/10.1089/ neu.2013.3162

Ghorbanpoor S, Garcia-Segura LM, Haeri-Rohani A, Khodagholi F, Jorjani M. Aromatase inhibition exacerbates pain and reactive gliosis in the dorsal horn of the spinal cord of female rats caused by spinothalamic tract injury. Endocrinology 2014; 155: 4341-55. https://doi.org/10.1210/en.2014-1158

Gonzalez SL, Coronel MF. Beyond reproduction: the role of progesterone in neuropathic pain after spinal cord injury. Neural Regen Res 2016; 11: 1238-40. https://doi. org/10.4103/1673-5374.189177

Honda K, Sawada H, Kihara T, Urushitani M, Nakamizo T, Akaike A, et al. Phosphatidylinositol 3-kinase mediates neuroprotection by estrogen in cultured cortical neurons. J Neurosci Res 2000; 60: 321-27. https://doi. org/10.1002/(SICI)1097-4547(20000501)60:3<321::AIDJNR6>3.0.CO;2-T

Honda K, Shimohama S, Sawada H, Kihara T, Nakamizo $\mathrm{T}$, Shibasaki $\mathrm{H}$, et al. Nongenomic antiapoptotic signal transduction by estrogen in cultured cortical neurons. J Neurosci Res 2001; 64: 466-75. https://doi.org/10.1002/ jnr. 1098

Ivanova T, Karolczak M, Beyer C. Estrogen stimulates the mitogen-activated protein kinase pathway in midbrain astroglia. Brain Res 2001; 889: 264-69. https://doi.org/10.1016/ S0006-8993(00)03149-8

Ivanova T, Mendez P, Garcia-Segura LM, Beyer C. Rapid stimulation of the pi3-kinase/akt signalling pathway in developing midbrain neurones by oestrogen. J Neuroendocrinol 2002; 14: 73-9. https://doi.org/10.1046/j.00071331.2001.00742.x

Ji RR, Gereau RWt, Malcangio M, Strichartz GR. Map kinase and pain. Brain Res Rev 2009; 60: 135-48. https://doi. org/10.1016/j.brainresrev.2008.12.011

Kawasaki Y, Kohno T, Ji RR. Different effects of opioid and cannabinoid receptor agonists on c-fiber-induced extracellular signal-regulated kinase activation in dorsal horn 
neurons in normal and spinal nerve-ligated rats. J Pharmacol Exp Ther 2006; 316: 601-7. https://doi.org/10.1124/ jpet.105.093583

Lee JY, Choi SY, Oh TH, Yune TY. 17beta-estradiol inhibits apoptotic cell death of oligodendrocytes by inhibiting rhoa-jnk3 activation after spinal cord injury. Endocrinology 2012; 153: 3815-27. https://doi.org/10.1210/en.20121068

Lewin GR, Ritter AM, Mendell LM. Nerve growth factor-induced hyperalgesia in the neonatal and adult rat. J Neurosci 1993; 13: 2136-48. https://doi.org/10.1523/JNEUROSCI.13-05-02136.1993

Lucas JM, Ji Y, Masri R. Motor cortex stimulation reduces hyperalgesia in an animal model of central pain. Pain 2011; 152: 1398-407. https://doi.org/10.1016/j.pain.2011.02.025

Masoudi A, Dargahi L, Abbaszadeh F, Pourgholami MH, Asgari A, Manoochehri M, et al. Neuroprotective effects of astaxanthin in a rat model of spinal cord injury. Behav Brain Res 2017; 329: 104-10. https://doi.org/10.1016/j. bbr.2017.04.026

Morissette M, Le Saux M, D’Astous M, Jourdain S, Al Sweidi S, Morin N, et al. Contribution of estrogen receptors alpha and beta to the effects of estradiol in the brain. $\mathrm{J}$ Steroid Biochem Mol Biol 2008; 108: 327-38. https://doi. org/10.1016/j.jsbmb.2007.09.011

Mosquera L, Colon JM, Santiago JM, Torrado AI, Melendez M, Segarra AC, et al. Tamoxifen and estradiol improved locomotor function and increased spared tissue in rats after spinal cord injury: Their antioxidant effect and role of estrogen receptor alpha. Brain Res 2014; 1561: 11-22. https://doi.org/10.1016/j.brainres.2014.03.002

Onose G, Anghelescu A, Muresanu DF, Padure L, Haras MA, Chendreanu CO, etal.Areview of published reports on neuroprotection in spinal cord injury. Spinal Cord 2009; 47: 716-26. https://doi.org/10.1038/sc.2009.52

Patel C, Xu Z, Shosha E, Xing J, Lucas R, Caldwell RW, et al. Treatment with polyamine oxidase inhibitor reduces microglial activation and limits vascular injury in ischemic retinopathy. Biochim Biophys Acta 2016; 1862: 1628-39. https://doi.org/10.1016/j.bbadis.2016.05.020

Ray SK, Samantaray S, Smith JA, Matzelle DD, Das A, Banik NL. Inhibition of cysteine proteases in acute and chronic spinal cord injury. Neurotherapeutics 2011; 8: 180-86. https://doi.org/10.1007/s13311-011-0037-1

Ren K. An improved method for assessing mechanical allodynia in the rat. Physiol Behav 1999; 67: 711-16. https://doi. org/10.1016/S0031-9384(99)00136-5
Ritz MF, Hausmann ON. Effect of 17beta-estradiol on functional outcome, release of cytokines, astrocyte reactivity and inflammatory spreading after spinal cord injury in male rats. Brain Res 2008; 1203: 177-88. https://doi. org/10.1016/j.brainres.2008.01.091

Saghaei E, Abbaszadeh F, Naseri K, Ghorbanpoor S, Afhami M, Haeri A, et al. Estradiol attenuates spinal cord injury-induced pain by suppressing microglial activation in thalamic vpl nuclei of rats. Neurosci Res 2013; 75: 316-23. https:// doi.org/10.1016/j.neures.2013.01.010

Samantaray S, Smith JA, Das A, Matzelle DD, Varma AK, Ray SK, et al. Low dose estrogen prevents neuronal degeneration and microglial reactivity in an acute model of spinal cord injury: Effect of dosing, route of administration, and therapy delay. Neurochem Res 2011; 36: 1809-16. https://doi.org/10.1007/s11064-011-0498-y

Samantaray S, Sribnick EA, Das A, Thakore NP, Matzelle D, Yu SP, et al. Neuroprotective efficacy of estrogen in experimental spinal cord injury in rats. Ann N Y Acad Sci 2010; 1199: 90-4. https://doi.org/10.1111/j.17496632.2009.05357.x

Sanna MD, Ghelardini C, Galeotti N. Activation of jnk pathway in spinal astrocytes contributes to acute ultra-low-dose morphine thermal hyperalgesia. Pain 2015; 156: 1265-75. https://doi.org/10.1097/j.pain.0000000000000164

Schaeffer V, Meyer L, Patte-Mensah C, Mensah-Nyagan AG. Progress in dorsal root ganglion neurosteroidogenic activity: basic evidence and pathophysiological correlation. Prog Neurobiol 2010; 92: 33-41. https://doi.org/10.1016/j.pneurobio.2010.04.009

Sezer N, Akkuş S, Uğurlu FG. Chronic complications of spinal cord injury. World J Orthop 2015; 6: 24-33. https://doi. org/10.5312/wjo.v6.i1.24

Singer CA, Figueroa-Masot XA, Batchelor RH, Dorsa DM. The mitogen-activated protein kinase pathway mediates estrogen neuroprotection after glutamate toxicity in primary cortical neurons. J Neurosci 1999; 19: 2455-63. https://doi. org/10.1523/JNEUROSCI.19-07-02455.1999

Sribnick EA, Samantaray S, Das A, Smith J, Matzelle DD, Ray SK, et al. Postinjury estrogen treatment of chronic spinal cord injury improves locomotor function in rats. $\mathrm{J}$ Neurosci Res 2010; 88: 1738-50. https://doi.org/10.1002/ jnr.22337

Sribnick EA, Wingrave JM, Matzelle DD, Wilford GG, Ray SK, Banik NL. Estrogen attenuated markers of inflammation and decreased lesion volume in acute spinal cord injury in rats. J Neurosci Res 2005; 82: 283-93. https://doi. 
org/10.1002/jnr.20622

Taves S, Berta T, Liu DL, Gan S, Chen G, Kim YH, et al. Spinal inhibition of p38 map kinase reduces inflammatory and neuropathic pain in male but not female mice: Sex-dependent microglial signaling in the spinal cord. Brain Behav Immun 2016; 55: 70-81. https://doi.org/10.1016/j. bbi.2015.10.006

Tohda C, Kuboyama T. Current and future therapeutic strategies for functional repair of spinal cord injury. Pharmacol Ther 2011; 132: 57-71. https://doi.org/10.1016/j. pharmthera.2011.05.006

Tozaki-Saitoh H, Tsuda M, Miyata H, Ueda K, Kohsaka S, Inoue $\mathrm{K}$. P2y12 receptors in spinal microglia are required for neuropathic pain after peripheral nerve injury. J Neurosci 2008; 28: 4949-56. https://doi.org/10.1523/JNEUROSCI.0323-08.2008

Wilson ME, Liu Y, Wise PM. Estradiol enhances akt activation in cortical explant cultures following neuronal injury. Brain Res Mol Brain Res 2002; 102: 48-54. https://doi. org/10.1016/S0169-328X(02)00181-X

Zhang H, Zhou F, Li C, Kong M, Liu H, Zhang P, et al. Molecular mechanisms underlying the analgesic property of intrathecal dexmedetomidine and its neurotoxicity evaluation: an in vivo and in vitro experimental study. PLoS One 2013; 8: e55556. https://doi.org/10.1371/journal.pone.0055556 